\title{
De la formación inicial al trabajo docente: Comprensión de la trayectoria ${ }^{[1]}$
}

Sánchez-Sánchez, Gerardo Ignacio; Jara- Amigo, Ximena Elizabeth

De la formación inicial al trabajo docente: Comprensión de la trayectoria [1]

Revista Educación, vol. 42, núm. 2, 2018

Universidad de Costa Rica, Costa Rica

Disponible en: http://www.redalyc.org/articulo.oa?id=44055139001

DOI: https://doi.org/10.15517/revedu.v42i2.23996

Esta obra está bajo una Licencia Creative Commons Atribución-NoComercial-SinDerivar 3.0 Internacional. 


\section{De la formación inicial al trabajo docente: Comprensión de la trayectoria ${ }^{[1]}$}

From initial teacher training to actual teaching work: understanding the trajectory

Gerardo Ignacio Sánchez-Sánchez 2

Universidad Católica del Maule, Chile

DOI: https://doi.org/10.15517/revedu.v42i2.23996

gsanchez@ucm.cl

Ximena Elizabeth Jara-Amigo 3

Universidad Autónoma de Chile, Chile

Recepción: 27 Abril 2016

xjaraa@uautonoma.cl

Aprobación: 05 Septiembre 2017

\section{Resumen:}

El presente artículo explora la comprensión de las experiencias pedagógicas en el contexto de la práctica profesional por parte de 32 docentes de distintas titulaciones. Los resultados evidencian una imagen de la docencia como un encuentro humano y no solo técnico, entre sujetos, contextos y demandas. Se comprende como una actividad dotada de complejidad en tanto tarea humana, y un proceso que se torna visible en el espacio de la práctica, la cual se constituye en fuente de aprendizaje y de crisis productiva. Se adoptó el enfoque cualitativo a partir de la modalidad de estudio de caso interpretativo con aplicación de entrevistas semiestructuradas, y una estrategia de discusión de resultados sustentada en el análisis de contenido. La investigación se desarrolló en el campus regional de una universidad, ubicada a 200 millas al sur de Santiago de Chile. El estudio abre perspectivas de diálogo en el contexto regional, susceptibles de enriquecer la formación inicial docente, al entender que no existen modelos ideales de profesorado al margen de los contextos o marcos de actuación que regulan el ejercicio de la docencia, por lo que se hace necesario sintonizar los modelos teóricos de formación con los límites y posibilidades que ofrece la práctica.

Palabras clave: Trabajo docente, educación primaria, práctica, Chile.

\section{Abstract:}

This article explores the understanding of pedagogical experiences of 32 teachers from different cohorts during a professional practicum. The results show an image of teaching as a human encounter, not only as a technical one, among subjects, contexts, and demands. It is understood as an activity endowed with complexity as a human task and a process that becomes visible within the practicum arena, which develops into a learning as well as a productive crisis source. The qualitative approach was adopted built on the interpretive case study modality; semi-structured interviews and a results discussion strategy based on content analysis were used. The research was conducted on the regional campus of a university, located 200 miles south of Santiago de Chile. The study opens up perspectives for dialogue within the regional context, having the potential to enrich initial teacher training, by means of understanding that there are no ideal models of teachers regardless of the settings or performing frameworks that regulate teaching; this makes it necessary to tune in theoretical training models to the limits and possibilities presented by the teaching practice.

KEYWORDS: Teachers work, primary education, practice, Chile.

\section{NOTAS DE AUTOR}

2 Profesor de Historia y Geografía. Magister en política y gestión educacional (Universidad de Talca). Doctor en Ciencias de la Educación (Universidad de la Serena). Se ha desempeñado como Director de Escuela de Pedagogía en Educación General Básica, Vicedecano de la Facultad de Educación y Director del Centro de Estudios Pedagógicos de la Universidad Autónoma de Chile. Actualmente se desempeña como académico adscrito al Proyecto de Mejoramiento institucional (PMI 1310) de la Universidad Católica del Maule - CHILE. Sus investigaciones en el ámbito de la formación inicial de profesores y procesos de práctica, han permitido publicar los siguientes artículos scielo: Visión del trabajo docente en el ámbito de la evaluación, que comienza a construir el profesorado en formación, a partir del uso de incidentes críticos en los procesos de formación práctica. Revista Electrónica Educare, Los espacios de tutoría en práctica profesional y sus necesidades de fortalecimiento. Revista Actualidades Investigativas en Educación y Visión del trabajo docente, de profesores en formación, a partir del uso de incidentes críticos. Revista Actualidades Investigativas en Educación.

3 Profesora de Historia y Geografía. Magister en Curriculum y Evaluación. Universidad Arcis. Doctora en Cultura y Educación en América Latina. Universidad Arcis. Se ha desempeñado como Coordinadora del área de prácticas de las escuelas de pedagogía en Historia, Geografía y Ciencias Sociales y pedagogía en Educación Básica de la Universidad Autónoma de Chile. Actualmente se encuentra adscrita a la Facultad de Educación de la Universidad Autónoma de Chile, Sede Talca - Chile, en calidad de Secretaria de Estudios de la Carrera de Historia, Geografía y Ciencias Sociales. Sus publicaciones scielo en el ámbito de la formación inicial de profesores son: Visión del trabajo docente en el ámbito de la evaluación, que comienza a construir el profesorado en formación, a partir del uso de incidentes críticos en los procesos de formación práctica. Revista Electrónica Educare, Los espacios de tutoría en práctica profesional y sus necesidades de fortalecimiento. Revista Actualidades Investigativas en Educación y Visión del trabajo docente, de profesores en formación, a partir del uso de incidentes críticos. Revista Actualidades Investigativas en Educación. 
La formación inicial docente constituye un proceso sistemático y organizado, orientado a proveer el desarrollo de competencias propias del ejercicio profesional, que integra el conocimiento disciplinario y pedagógico, además de las habilidades o estrategias necesarias, y las cualidades personales y sociales requeridas para el trabajo docente (Cornejo y Fuentealba, 2008).

La creciente complejidad social que caracteriza el contexto actual determina, en consecuencia, una formación docente más compleja, que deberá responder a estándares pedagógicos y disciplinarios de mayor exigencia. En ese escenario, los modelos de formación inicial de profesorado descubren, en la práctica, un espacio de potencialidad formativa si esta logra proporcionar "oportunidades para asumir un rol protagónico y, desde allí, comprender y desarrollar las competencias involucradas en el ejercicio profesional" (Montecinos, Solís, Contreras y Rittershaussen, 2009, p. 47).

Un programa de formación inicial que intente relevar la práctica requiere relaciones significativas entre los centros educativos, las universidades y los profesorados en formación, comprendido desde tres perspectivas simultáneas: de la persona que aprende a enseñar, de cada docente de aula que colabora en el proceso formativo y de la persona formadora de profesorado desde la universidad.

El modelo de formación de profesorado de la Facultad de Ciencias de la Educación de la Universidad Católica del Maule define una línea de práctica que constituye un eje horizontal y progresivo de inserción del futuro profesorado en los centros educativos. Desde el inicio de su formación y con un carácter progresivo, su propósito es el desarrollo de las competencias profesionales de un personal docente reflexivo, crítico e investigador de su quehacer. Esta línea de práctica procura oportunidades para que el profesorado en formación vaya asumiendo, paulatinamente, roles protagónicos como sujeto y objeto de análisis crítico, promoviendo el cuestionamiento de la teoría a partir del enfrentamiento con problemas educativos.

El eje de práctica contempla distintas experiencias para que, de manera progresiva, los grupos profesionales en formación desarrollen las competencias necesarias que les permitan generar aprendizajes efectivos en el alumnado que atienden, reflexionando sobre diversas situaciones en el campo laboral desde la perspectiva de "aprender a enseñar", basada en un enfoque socio constructivista. Para ello, el estudiantado participará en una serie de experiencias de acercamiento a realidades laborales diversas, organizadas en cuatro etapas: (1) Prepráctica, (2) Práctica I, (3) Práctica II y (4) Acompañamiento al titulado (Universidad Católica del Maule, 2015).

En ese contexto se inscribe la investigación Visión del trabajo docente desarrollado por estudiantes en procesos de inserción al aula, desarrollada en el contexto de la Facultad de Ciencias de la Educación entre el 2015 y 2016, y que considera relevante detenerse en el proceso que experimenta el grupo profesional en formación en su inserción en los territorios escolares, por lo cual indaga en la comprensión situacional que, desde la práctica y desde una perspectiva deliberativa y fenomenológica, desarrolla de la docencia y el trabajo docente.

El artículo que se presenta tiene como objetivo explorar la comprensión de las experiencias pedagógicas que comienza a vivir el profesorado en formación en su trayecto por la práctica, profundizando particularmente en el tipo de docente que es, siente o busca ser, y en los principales obstáculos, problemas o limitaciones que debe enfrentar para concluir satisfactoriamente su proceso formativo. Se asumió la investigación con un abordaje cualitativo.

\section{REFERENTES CONCEPTUALES}

El cuestionamiento actual planteado al desempeño del profesorado y el surgimiento de nuevas perspectivas educativas ponen de manifiesto la necesidad de remirar la formación inicial, entendiendo el desafío de vincular ámbitos y contextos de naturaleza compleja -la teoría y la práctica; la universidad y el mundo de la escuela; enseñanza y aprendizaje- y, por tanto, asumir e el imperativo de superar el desfase habitual entre una 
formación teórica normativa y desvinculada de la realidad, y una práctica muchas veces de sentido común que responde reactivamente a la incertidumbre y necesidades del aula.

Tarea no exenta de dificultad, pues en lo referido a los procesos de práctica, los aspectos críticos relevados por la investigación ponen de manifiesto la inadecuada comprensión de la naturaleza teórico- práctica de la acción pedagógica (Cisternas, 2011; Labra, 2011; Solís, M., Núñez, C., Contreras, I., Rittershaussen, Montecinos, y Walker, 2011; Ávalos y Matus, 2010; Ortúzar, Flores, Milesi y Cox, 2009; Latorre, 2006; 2009; Montecinos, C. y Walker, H., 2010; Montecinos, C., Barrios, C. y Tapia, M., 2011; Nocetti, A., Mendoza, M., Contreras, G., Sanhueza, K. y Herrera, S., 2005; Ritterhaussen, S., Contreras, I., Suzuki, E., Solís, M., y Valverde, P., 2004). La evidencia empírica aportada por estos estudios permite constatar que la implementación de los espacios y procesos de práctica se desarrollan como actividad de transmisión de datos, en general, de manera descontextualizada; caracterizada por el divorcio del proceso formativo respecto de los reales requerimientos de los contextos escolares. De ahí la necesidad de profundizar en este escenario, para retroalimentar el proceso formativo del profesorado en formación, y de esa forma avanzar a la implementación de una práctica comprendida como una actividad reflexiva y fuertemente contextualizada.

Tarea no exenta de dificultad, pues en lo referido a los procesos de práctica, los aspectos críticos relevados por la investigación ponen de manifiesto la inadecuada comprensión de la naturaleza teórico- práctica de la acción pedagógica (Cisternas, 2011; Labra, 2011; Ávalos y Matus, 2010; Ortúzar, Flores, Milesi y Cox, 2009; Latorre, 2006; 2009; Montecinos, C. y Walker, H., 2010; Montecinos, C., Barrios, C. y Tapia, M., 2011; Nocetti, A., Mendoza, M., Contreras, G., Sanhueza, K. y Herrera, S., 2005; Ritterhaussen, S., Contreras, I., Suzuki, E., Solís, M., y Valverde, P., 2004). La evidencia empírica aportada por estos estudios permite constatar que la implementación de los espacios y procesos de práctica se desarrollan como actividad de transmisión de datos, en general, de manera descontextualizada; caracterizada por el divorcio del proceso formativo respecto de los reales requerimientos de los contextos escolares. De ahí la necesidad de profundizar en este escenario, para retroalimentar el proceso formativo del profesorado en formación, y de esa forma avanzar a la implementación de una práctica comprendida como una actividad reflexiva y fuertemente contextualizada.

Ser parte de un proceso de formación inicial de docentes, sustentado en la realidad de las escuelas y comunidades concretas en las que se incorporan y volver a estos mismos espacios aúlicos para experimentar nuevas formas de actuar y reflexionar, permite apropiarse de los contextos de ejercicio de la profesión y de la naturaleza que caracteriza el trabajo docente. Necesitamos una formación inicial "que debe realizarse partiendo y volviendo a la práctica en un proceso que contemple el estudio, la reflexión, la discusión, la experimentación" (Imbernon, 1994, p. 60).

En ese escenario, nos enfrentamos a dos modelos de formación: técnico y crítico reflexivo. En el primer modelo, el foco se encuentra en "la transmisión de conocimientos científicos y culturales, para dotarles de una formación especializada, centrada, principalmente, en el dominio de los conceptos y la estructura disciplinaria de las materias de las cuales será especialista” (Cornejo y Fuentealba, 2008, p.14), concibe la práctica como espacio de aplicación del conocimiento. En esa dirección, " un profesor ha de ser formado como un técnico competente, sobre todo en la planificación de su actividad docente, de modo que pueda ser adecuadamente evaluada la eficacia de su trabajo" (Rozada, 1997, p. 59).

La preeminencia de este enfoque arroja, como problema sustantivo, el desconocimiento de los problemas de la práctica docente como complejos e indeterminados, pues "involucran a sujetos y procesos culturales" (Davini, 1995, p. 105). Siguiendo al autor, "la perspectiva de la práctica restringida al aprender a enseñar en el aula tiende a reducir el trabajo docente a su dimensión técnica, desestimando sus dimensiones sociocultural y ético-política" (p. 114).

En el segundo modelo, asumiendo una mirada reflexiva, el foco de aprendizaje está centrado en la experiencia y en la observación. Esta experiencia y su potencialidad "no ocurre en el vacío, sino que en interacción con sujetos que ya traen esquemas conceptuales previos” (Cornejo y Fuentealba, 2008, p.14); 
ello implica entender que la enseñanza se desarrolla en contextos de complejidad creciente, que obligan a una relación distinta teoría - práctica.

El desafío de la formación inicial pasa por superar el efecto simplificador que impone la visión de procesos eficientes centrados en la transmisión de un saber instrumental, donde la inmersión temprana al aula tiende a constituir un proceso de aprendizaje de naturaleza adaptativa a las reglas de juego derivadas de la enseñanza, el cual desaprovecha la posibilidad de concebirla como un verdadero proceso de aprendizaje de la naturaleza de la profesión (Davini, 1995), transida de una compleja trama de relaciones entre los sujetos y sus contextos.

Esta última idea viene a confirmar el hecho, según el cual, el aprendizaje de la profesión docente refiere a un trabajo tensionado por acciones de naturaleza técnica, afectiva, ético-política e interactiva que se deben desarrollar en los espacios escolares, vale decir, supone "la presencia simultánea y necesaria de distintos tipos de acción, que obligan a los actores a realizar una gran variedad de interacciones con los alumnos en función de varios objetivos que no son necesariamente coherentes u homogéneos" (Tardif, 2004, p. 129).

Desde la perspectiva de este autor, el trabajo docente es, fundamentalmente, un conjunto de interacciones personalizadas con estudiantes para conseguir su participación en su proceso formativo, un trabajo que exige una inversión afectiva y cognitiva profunda, según la cual se "debe también empeñar e invertir en ese trabajo lo que él mismo es como persona" (Tardif, 2004, p. 104).

En consecuencia, para formar en una perspectiva más en sintonía con la realidad, es preciso avanzar hacia una racionalidad que se haga cargo de la complejidad que supone la docencia, en lo que Diker y Terigi (1997) denominan certeza situada y que desde la perspectiva de Baillauques (2010) implica entender la necesidad de formar, por una parte, un sujeto con apertura, flexibilidad y autonomía para enfrentar los contextos; y por otra, de estructura, aceptación y adaptación a las reglas sociales definidas en términos de resultados esperados.

El espacio que está llamado a facilitar la formación y una adecuada comprensión de la naturaleza del trabajo docente es el de la práctica. Una práctica como lugar de constante producción de nuevas soluciones a los problemas con los cuales se ven enfrentados los profesionales. Coherente con este requerimiento y de acuerdo con lo planteado por Carbonneau y Hétu, (2010, p.129), la práctica debería suscribir los siguientes principios de actuación: "acelerar la alternancia entre la formación para el análisis conceptual y la formación para la intervención; multiplicar las situaciones y modelos pedagógicos y fundamentalmente valorar la toma de conciencia del profesor en formación", esto último en lo referido a las concepciones y esquemas de acción que va desarrollando en sus procesos de inserción al aula.

Al enfrentar los requerimientos de la práctica, cada estudiante de pedagogía desarrolla distintas representaciones sobre lo que puede ser su aterrizaje al interior de una sala de clases. De hecho, enfrenta la práctica a partir de ciertos proyectores conceptuales e imágenes previas a la formación inicial, desarrollando una percepción aún incompleta de la realidad para navegar en el territorio del aula, donde constata que nada o muy poco se comporta como estaba previsto. Se ve necesitado de reglas flexibles y cambiantes, pues la explicación teórica omnisciente no le sirve o no le brinda todas las respuestas. Ello implica que "una formación profesional que gire, en un principio, en torno a la comprensión y al análisis teórico de la intervención contribuye a retrasar la aprehensión de la economía pedagógica por incitar a realizar una lectura fragmentaria de las situaciones" (Carbonneau, y Hétu 2010, p. 128).

Es importante entender que el profesorado en formación experimenta la inserción a la práctica y al trabajo docente enfrentando el desafío de otorgar sentido a su experiencia pedagógica, partiendo de la base de que "el rol de las teorías, las prescripciones externas y las experiencias de otros docentes adquiere relevancia cuando ellas pueden articularse con la experiencia del docente y, a la vez, tenga sentido en su trabajo" (Fullan y Hargreaves, 1996, p. 178).

En la búsqueda de este sentido, el profesorado se encuentra continuamente ocupado en aprender más sobre sí mismo y su manera de trabajar, desarrollando sus propias comprensiones del proceso. De ahí la importancia de detenerse en la comprensión de las experiencias pedagógicas que vive el profesorado en formación, como 
una forma de identificar los procesos que constituyen el aprender a enseñar y las categorías conceptuales en las que articula el conocimiento para desarrollar la actividad profesional de la enseñanza.

\section{Procedimientos metodológicos}

Este artículo da cuenta de la investigación Visión del trabajo docente desarrollada por estudiantes en procesos de inserción al aula (Sánchez, 2015 y 2016), en el contexto de un programa de formación de profesorado de educación primaria en una universidad regional.

Es un estudio de alcance descriptivo que utiliza un enfoque de investigación cualitativa (Hernández, Fernández y Baptista, 2010).

El trabajo, desde el punto de vista metodológico, asume la modalidad de estudio de casos de carácter interpretativo (Pérez, 2007), pues centra su atención en descripciones ricas y densas, las que son empleadas para elaborar categorías conceptuales o para ilustrar, defender o desafiar presupuestos teóricos sostenidos antes de recoger los datos.

A partir de los criterios de selección planteados por Flick (2007), se utilizan los siguientes criterios de selección de la muestra de estudiantes en práctica: optar por casos típicos, apuntando a develar el campo desde dentro y desde su centro; optar por una selección según intensidad con la que los rasgos, los procesos, las experiencias, etc., interesantes se producen; e incluso seleccionar casos que sean de más fácil acceso (conveniencia).

La recolección de información se lleva a cabo a través de entrevista semiestructurada sustentada en un guión de preguntas que indagan en torno a la percepción sobre la docencia en contextos de práctica. Más específicamente, se consulta al profesorado en formación sobre el tipo de docente en el que pretende convertirse a partir de lo experimentado en su proceso de práctica, y se indaga en las dificultades, problemáticas y obstáculos que comienzan a vivenciar en el trabajo de aula. Haciendo uso de un protocolo que implicó el uso del consentimiento informado, el instrumento fue administrado a 32 estudiantes de las menciones de lenguaje, matemática, ciencias sociales y ciencias naturales que experimentan su práctica profesional en establecimientos de educación primaria. Dicha muestra se compone de 10 docentes de lenguaje, 8 de matemática, 7 de ciencias naturales y 7 de ciencias sociales. Respecto a las personas participantes, el $62.5 \%$ corresponde a mujeres y un $37.5 \%$ a hombres. Sus edades fluctúan entre 24 y 27 años. Estos profesores forman parte de un programa de pedagogía básica con mención en una institución universitaria financiada con fondos públicos.

Una vez recogida la información, se procede a la transcripción de las entrevistas y a la preparación del material para su análisis. Posteriormente, haciendo uso del análisis de contenido, se levantan de manera inductiva las dimensiones y categorías que se analizan y ejemplifican con citas textuales para profundizar en la comprensión de las experiencias pedagógicas que, en el contexto de inserción al aula, desarrollan el profesorado en formación.

\section{Resultados}

En primer lugar, se presentan los resultados tendientes a develar las comprensiones que elabora el profesorado en formación sobre el tipo de docente en el que aspira convertirse. En segundo lugar, se explicitan los obstáculos, problemas o limitaciones que enfrentan en su inserción a la práctica en aulas reales. En ambos casos, se trabaja a partir de las dimensiones y categorías provenientes del análisis de contenido de las entrevistas realizadas. 


\subsection{Qué tipo de docente soy o me siento o busco ser (Ver Tabla 1)}

TABLA 1

Tipo de docente que soy - me siento o busco ser

\begin{tabular}{ll}
\hline Dimensión & Categorías \\
\hline Ilustrado & Siempre informado \\
& Saber lo que estoy enseñando \\
\hline Técnico & Buen trabajo: relacionando contenidos y reglas claras \\
& Detector de necesidades \\
& Eficiente y eficaz \\
\hline Practicante artesano & Puesto por puesto, conociéndolos \\
& Descubrir y hacer \\
& Contextualización \\
& Captar la fibra íntima de cada curso \\
\hline Practicante reflexivo & Pedagogía comprensiva \\
& Autorregulación \\
& Consciente de mis capacidades \\
& Conductista / constructivista \\
\hline Actor social & Democrático \\
& Utilidad para la vida - transversalidad \\
& Empatía social - compromiso \\
& El mercado gobierna las profesiones \\
\hline Cencano al alumnado \\
Ser ejemplo - respetado \\
Involucrado \\
Consecuente \\
Equilibrado \\
Busco ser y creo ir \\
Tarea tan humana \\
\hline
\end{tabular}

Nota: Elaboración propia (2016).

Las comprensiones de los sujetos entrevistados se muestran en un crisol que integra distintas facetas del rol: profesorado ilustrado, técnico, practicante artesano, reflexivo, actor social y persona.

ROL ILUSTRADO. Esta dimensión emerge en la idea de un profesorado conocedor de su ámbito disciplinario, aspecto considerado clave para sobrellevar su labor pedagógica. A continuación, se presentan algunas respuestas que ponen de manifiesto las distintas categorías que surgen de las respuestas de los sujetos:

"Me gustaría ser una maestra que domine con exactitud todas las disciplinas, una persona siempre informada presentando conocimientos generales de actualidad”. (BH) 
"Que maneja los contenidos que enseña, particularmente cuando nos corresponde la enseñanza en distintos niveles". (FC)

ROL TÉCNICO. La dimensión técnica asociada a la docencia refiere a la idea de un profesorado que posee las habilidades necesarias para lograr una actuación eficaz en el aula, en coherencia con los objetivos definidos. Las siguientes respuestas permiten ilustrar la dimensión:

"Busco ser un profesor equilibrado entre el aprendizaje de contenidos y valores, no busco hacer cambios rupturistas, ni tampoco buscar una utopía educativa, sino que hacer un buen trabajo de resultados estandarizados”. (LC)

"Soy eficaz y eficiente al momento de realizar clases, evaluaciones y después para revisar, evito dejar trabajo para más tarde para no verme atrasado y estresado por lo mismo reconozco que me gusta tener el control del aula, pues he aprendido que una cosa es la planificación de la clase, y otra es la destreza para llevarla a cabo”. (RC)

ROL PRACTICANTE ARTESANO. Se plantea la idea de un profesorado que adquiere los trucos del oficio en la práctica, por lo que finalmente la práctica y el espacio del aula adquieren más importancia que la formación teórica.

Es reconocido como el tipo de docente con preocupación por conocer a sus estudiantes, a quienes considera en su diversidad y desde ahí enfoca los esfuerzos que faciliten el aprender. Las siguientes respuestas ponen de manifiesto esta faceta de la tarea docente:

“El que va puesto por puesto conociéndolos y desde ahí buscando la mejor forma de generar aprendizajes”. (CAC)

"Quiero ser la profesora que se preocupe de que sus alumnos aprendan, pero no de la forma tradicional, sino que aprendan interactivamente en las clases para que los alumnos cambien su mentalidad y dejen de asociar el aburrimiento al aprendizaje”. (NI)

En ese sentido, emerge la idea de una persona profesional que, en el hacer del aula, va descubriendo claves propias y singulares de actuación. Más aún cuando se reconoce fuertemente la condición de novatez que las siguientes respuestas ponen de relieve:

"Creo que soy una docente novata, que requiero y necesito mucha experiencia, creo que hay mucho por descubrir y por hacer, siento que soy una docente consciente de lo que se espera de mi profesión y de la responsabilidad que he tomado por decisión propia”. (AC)

"Hoy en día soy un profesor novato enfrentado a una realidad que sobrepasa lo pensado en la universidad”. (PC)

Con buen ojo observador del espacio donde se sitúa el proceso de enseñanza- aprendizaje, tiene el llamado a comprender la importancia de la acción pedagógica contextualizadora, como se refleja en los siguientes relatos:

Busco ser y he tratado mucho, de ser innovadora, creativa y, sobre todo, contextualizadora, creo que los alumnos aprenden más cuando entienden el por qué están aprendiendo tal contenido y la utilidad que tiene para su vida y pueden reflexionar sobre ello. También creo aprenden más con experiencias que les agradan, que les son cercanas y llamativas, además busco ser cercana a ellos y poder conocerlos". (AC)

"Busco ser y creo ir en el camino de ser un profesor que trata de dar utilidad real a lo que enseña; capaz de captar la fibra de cada curso, que realimente constituye el gran desafío". $(\mathrm{OH})$

ROL PRACTICANTE REFLEXIVO. Surge la noción de un profesorado capaz de analizar sus propias actuaciones, de resolver problemas y de inventar estrategias que respondan a las necesidades de sus estudiantes y los requerimientos contextuales.

Lejos de la pedagogía como acción técnica, se aproxima a imágenes más deliberativas y comprensivas del trabajo docente, como bien lo reflejan los relatos que a continuación se presentan:

"Busco en realidad ser distinta, trabajar en una pedagogía más comprensiva, cercana, conciliadora”. (RH) 
"Hoy en día soy un profesor novato que intenta entender aún el sistema educacional y busco ser un profesor capaz en todos los sentidos, lo que me obliga a detenerme en lo que hago, observando mi práctica”. (PC)

Este profesorado en formación, a través de su proceso de observación, acción y reflexión, toma distancia respecto a la figura autoritaria de encuentro docente- estudiante, aun cuando enfrentan las tentaciones de dejarse atrapar por miradas excluyentes o dicotómicas que suelen estar representadas por el conductismo y constructivismo. Ello queda de manifiesto en el siguiente relato:

"Me gustaría que el trabajo en la asignatura sea una labor en conjunto con mis estudiantes, haciéndolos partícipes de ello y no yo solo figurar como la persona autoritaria que sabe absolutamente todo, que me haga crecer a mí como persona y profesional y que cada experiencia me entregue las herramientas necesarias para seguir mejorando". (JH)

Particularmente, concibe su acción en términos de autorregulación, ayudando, por una parte, a sus estudiantes a ser más consciente de su proceso formativo, y a ellas o ellos mismos, a comprometerse con la necesidad de revisión de lo que se hace y de las opciones que se asumen, lo que les obliga a tomar conciencia de las capacidades propias, desde las cuales se piensa, actúa y valora. Así lo reflejan los relatos siguientes:

"Busco ser un docente que logre cumplir con las exigencias del mundo de hoy, un profesor que logre abrir los ojos de sus estudiantes y que los inste a buscar el conocimiento". (FC)

"Pretendo ser un profesor capaz de analizar mi práctica, me considero una persona reflexiva". (IC)

ROL CENTRADO EN LA PERSONA. Esta dimensión evidencia la idea de un profesorado que es ante todo persona, y que vive el proceso de inserción al aula, siendo él mismo un instrumento clave del trabajo que desarrolla. Este profesorado en formación, busca ser profesional cercano a sus estudiantes, pues reconoce que ante todo se trabaja con personas a las cuales es preciso tratar en coherencia. Así lo muestran los relatos que se presentan a continuación:

"El profesor que pretendo ser es un profesional cercano a los estudiantes, es un trabajo, pero ante todo se trabaja con personas y a las cuales es preciso respetar". (DC)

"Me siento un profesor cercano, que maneja los contenidos que enseña con ganas de dialogar y conocer siempre a mis alumnos para transformar aunque sea de una forma pequeña su proceso de aprendizaje”. (FC)

Se declara consciente de que sus juicios y acciones deben permitirle constituirse en ejemplos para sus estudiantes, como se advierten en las siguientes respuestas:

\footnotetext{
"Busco ser un docente que con el ejemplo, muestre que la cultura y la lectura son dominio que marquen entre un ser que piensa y que no, que logre contribuir a la emancipación de los jóvenes a las ataduras del siglo XXI, del consumo, de la ignorancia, la idea es hacerles ver lo engañados que estamos en esta época y esa necesidad de comprender todos los sesgos ideológicos explícitos e implícitos”. (GC)

"Busco ser una docente que deje huellas positivas en mis alumnos, ser un modelo a seguir por medio de mis juicios y actitudes". (KC)
}

Como toda relación humana, este profesorado en formación considera que la pedagogía lo desafía a involucrarse con sus estudiantes, para provocar el compromiso de estos grupos con el aprendizaje. Destaca el carisma en su relación con el estudiantado, como una condición para posibilitar el aprendizaje. Carisma que debe ir acompañado por la búsqueda de la consecuencia entre los juicios y acciones evidenciada en el trabajo de aula. Las respuestas presentadas a continuación respaldan sus comprensiones:

"Me involucro mucho en lo que les gusta o les llama la atención, me importa muchísimo que aprendan, busco y agoto la mayor cantidad de herramientas para lograr esto porque no me gusta la ignorancia, sé que no lo podemos saber todo, pero sé que por lo menos podemos intentarlo". (CC) 
"En consecuencia, ser un profesor carismático con ganas de enseñar, pero con una profunda humildad de estar perfeccionándose día a día para poder ayudar a los otros, es decir a los estudiantes que están en crecimiento y desarrollo”. $(\mathrm{AH})$

"Soy un profesor consecuente y consciente al momento de llevar a cabo mis clases, busco conocer a mis estudiantes en torno a una observación y análisis de su comportamiento y actuar en clases”. (SH)

Advierte, por otra parte, que el trabajo docente representa un proceso inacabado y, en consecuencia, constituye un desafío a permanecer en búsqueda permanente, como lo muestran las siguientes respuestas:

"Pero me doy cuenta que nunca se termina de aprender tanto en el aspecto cognitivo, emocional, psicológico, espiritual, social y ético". (BH)

"En la actualidad aún estoy en busca de aquella identidad, sin embargo, mis directrices buscan ser un profesor modelo para los estudiantes que no solo se enfoquen en el contenido teórico. Sino que reforzar en ellos otras áreas que les puedan ser útiles para sus vidas cotidianas". (JH)

Finalmente, percibe la docencia como un trabajo que al igual que la vida, conforma una tarea humana con la misma riqueza y complejidad.

"Quiero ser un buen docente, que logre el fin de éste, es decir, el aprendizaje de los jóvenes y que los alumnos deseen aprender autónomamente, pero también quiero ser amena, sensible siempre, innovadora, flexible, acogedora. A veces el cariño gana batallas sobre todo en esta tarea tan humana como es la docencia”. (VC)

"Creo que soy una docente novata, necesitada de conocimientos y experiencia, siento que soy una docente consciente de lo que se espera de mi profesión y de la responsabilidad que he tomado por decisión propia”. (MJC)

ROL ACTOR SOCIAL. La comprensión referida a esta dimensión surge anclada a la idea de un profesorado consciente y comprometido con una tarea cuya misión es transformadora y colectiva. Ello es reconocido en expresiones que refuerzan una serie de características sociales de la profesión, tales como su carácter democrático, de compromiso social, de apertura y utilidad en tanto se conecta con la vida, y consciente de la racionalidad del contexto social donde se desarrolla.

En su desafío social, el profesorado en formación pretende ser docente con capacidad de generar inquietud en sus estudiantes, con habilidad para comprender la realidad en que viven y, en su acción docente, procurar utilidad de aquello que enseña. Las siguientes respuestas así lo dejan planteado:

"Busco ser y creo ir en el camino de ser un profesor que trata de dar utilidad real a lo que enseña". (OH)

"Quiero esforzarme por entregarles herramientas para defenderse en la vida, siempre les hice la conexión que todos los procesos violentos de la historia fueron producto de la falta de empatía, de respeto, de sinceridad y confianza”. (JH)

Una parte se representa en la idea de constituir un profesorado comprometido con la transformación social, que busca transmitir esa conciencia y sensibilidad a sus estudiantes en el trabajo de aula. Así queda reflejado en sus respuestas:

"Yo creo ser una docente con convicciones claras, pero me doy cuenta que nunca se termina de aprender tanto en el aspecto cognitivo, emocional, psicológico, espiritual, social y ético. Empatía social expresada en el compromiso social con la formación de las futuras generaciones, conocimientos y habilidades prácticas que me permiten hacer entendible a la realidad contextual los contenidos". (BH)

"Busco ser un docente que logre cumplir con las exigencias del mundo de hoy, un profesor que logre abrir los ojos de sus estudiantes y, que logre contribuir a la emancipación de los jóvenes a las ataduras del siglo XXI, del consumo, de la ignorancia”. (GC)

Se declara consciente de las contradicciones que rodean al proceso educativo, dada la existencia de un modelo social y económico que parece operar con lógicas distintas, y es lo que manifiesta el siguiente relato: 
"El problema está en que no me preocupa el que no aprende. Avanzo con los que me siguen en las clases, el mercado gobierna hasta las profesiones más sociales e idílicas como supuestamente es la pedagogía, en tanto formación de personas". (JH)

En consecuencia, sobre la consulta respecto al tipo de docente en el que creen se están convirtiendo o busca ser, emerge la idea de un trabajo que muestra distintas aristas, en términos de las habilidades que demanda de sus agentes. Diversidad que se explica por el hecho de que los objetivos escolares, aparte de numerosos y variados, se suelen mostrar heterogéneos e inclusive poco coherentes. La consecuencia es el surgimiento de un trabajo que exige iniciativa docente, con un rol que tienen que interpretar y adaptar constantemente a los contextos diversos de la acción pedagógica, recurriendo en dicha tarea a diversos modelos o enfoques.

\subsection{Los obstáculos, problemas o limitaciones que he debido enfrentar para concluir satisfactoriamente el proceso formativo}

En la consulta por los obstáculos, problemas o limitaciones que ha debido enfrentar en su inserción, estos son clasificados en personales, pedagógicos, institucionales, sociales, vinculados a la formación recibida y al perfil del estudiante adolescente (Ver Tabla 2). 
TABLA 2

Obstáculos, problemas o limitaciones a enfrentar

\begin{tabular}{|c|c|}
\hline Dimensión & Categorías \\
\hline \multirow[t]{4}{*}{ Personales } & Inseguridad - temor \\
\hline & Que me vieran como docente \\
\hline & Yo soy mi única limitación (la mente propia) \\
\hline & Autodisciplina \\
\hline \multirow[t]{5}{*}{ Pedagógicos } & Disciplina y orden -manejo \\
\hline & Desmotivación estudiantil \\
\hline & Momentos de aula \\
\hline & Escaso impacto en el aprendizaje \\
\hline & Calificación y valor de cambio \\
\hline \multirow[t]{5}{*}{ Institucionales } & Interrupciones al proceso y aula \\
\hline & Cultura escolar \\
\hline & Evaluar valor de uso \\
\hline & Proyecto educativo institucional sin línea central \\
\hline & Diversidad de docentes en los centros \\
\hline \multirow[t]{3}{*}{ Perfil estudiantil } & Idiosincrasia de jóvenes \\
\hline & Atención y concentración \\
\hline & Responder a diversidad \\
\hline \multirow[t]{4}{*}{ Formación } & Peso de 12 años de escolaridad \\
\hline & Falta de acompañamiento \\
\hline & Sentirse sin protección \\
\hline & Replicar modelos \\
\hline \multirow[t]{3}{*}{ Sociales } & Olvido de valores \\
\hline & Énfasis en los resultados \\
\hline & Creencias y modelos instalados \\
\hline
\end{tabular}

Nota: Elaboración propia (2016).

\section{PERSONALES}

Dentro de las dificultades personales que condicionan su visión del trabajo docente, están las referidas a inseguridad que les genera disponer de las competencias requeridas, dada las características de estudiantes con los cuales se ha de encontrar. Inseguridad traducida en el miedo a no ser capaz de responder y que pone de manifiesto la necesidad de enfrentar los propios temores. Ello queda en evidencia en los relatos siguientes:

"Me enfrento diariamente con mi inseguridad, que, aumenta mi nerviosismo y me hace titubear en los contenidos". (CC)

"Soy muy olvidadiza puesto que, siempre tengo en cuenta muchas cosas y suelo olvidar las tareas y/o actividades a mediano y largo plazo, siempre trato de responder con las tareas más apuradas a corto plazo. Debo enfrentar mis miedos en cuanto 
a mis conocimientos que siempre creo que no son suficientes al enfrentar a un grupo curso, y pienso que no voy a tener el tiempo de estudiar de nuevo y ser suficientemente buena docente en cuanto a la parte cognitiva". (BH)

Asimismo, gran cantidad de este profesorado en formación enfrenta el trabajo docente reconociendo el temor a innovar, lo cual asocian fuertemente al peso de las experiencias preformativas que portan consigo y que los lleva a circunscribirse a esquemas más tradicionales de actuación. Ello se manifiesta en la respuesta presentada a continuación:

"Y creo que el peso de mis 12 años de escolaridad me jugó también en contra, ya que a veces me pillaba realizando cosas que sabía que no son las más esperadas. La creatividad es una cualidad que también me gustaría fomentar para mejorar mis clases futuras". (VC)

Dentro de sus percepciones, también emergen dificultades propias en términos de la inseguridad que provoca que sus estudiantes les vean como docente, y no tan solo un estudiante en práctica:

Una dificultad fue el lograr que los estudiantes me vieran como docente y no como una simple alumna en práctica, ya que ellos se justificaban en esto para tomarse las actividades de las clases más a la ligera. (MJC)

Concluye que gran parte de las limitaciones que enfrenta están asociadas a su propia persona, y reconoce como única limitación (la mente propia) que los lleva a dudar de las capacidades que posee para enfrentar los retos del trabajo docente, específicamente en relación con un perfil de estudiantes que ven, en lo particular, desafiante. Bien lo manifiestan los próximos relatos:

"La limitación principal es la mente propia ya que siempre está dudando de todo, cambia de parecer constantemente, va mutando muy rápido, lo que no permite adoptar una línea pedagógica con seguridad”. (GC)

"He llegado a la conclusión que yo soy mi única limitación en mi tarea de ser profesor". (FC)

\section{PEDAGÓGICOS}

Dentro de las dificultades pedagógicas, identifican especialmente las vinculadas con la generación de un clima propicio y el mantener una enseñanza para el aprendizaje de todo el grupo estudiantil. Así es como plantean los desafíos que encierran el tema de la disciplina y el orden; y asociada a este, el manejo de grupo.

"Los principales obstáculos han sido relacionados con el manejo de grupo, si bien se logra, a veces los alumnos no están dispuestos a hacer las cosas y se hace complejo pasar llamando la atención constantemente, diciendo que trabajen o que guarden silencio". (MAC)

Las deficiencias en cuanto al dominio de grupo, este hecho me frustraba un poco, ya que utilizaba actividades llamativas, lúdicas e incluso cortas para mantenerlos activos además no me caracterizaba, por ser alguien con falta de carácter. Por ejemplo, si tenía que llamar la atención a un alumno, lo hacía con un tono de voz fuerte y claro". (LC)

Manejo de grupo que se vincula con el reconocimiento de la desmotivación generalizada de estudiantes, y que obliga a invertir tiempo en conocerles para comprender su falta de interés por aquello que sus docentes les ofrecen en términos de oportunidades de aprendizaje. La respuesta siguiente lo respalda:

"Pese al buen comportamiento de los jóvenes, creo que la mayor limitación es su desmotivación y poco interés. No obstante, la limitación tal vez está en mí, puesto que no he sido capaz de innovar y generar algo que llame su atención”. (RC)

Otro grupo de dificultades se relaciona con el manejo y administración de la clase. Constata la existencia de problemas para articular los tiempos de la clase, pues en su implementación esta tarea se encuentra fuertemente condicionada por el comportamiento estudiantil y las respuestas que dan. Los siguientes relatos lo dejan demostrado:

"En ocasiones se torna complejo manejar los tiempos de la clase, pues la disposición de los estudiantes en variada, las interrupciones que se produce durante la clase, obligan a desarrollar la capacidad para retomarla y eso complica. Habitualmente no alcanzo al cierre de las clases y en ocasiones los contenidos no se abordan debiendo retomarlos en la clase siguiente". (PC) 
Generalmente comienzo bien mis clases sin embargo al final me cuesta poder centrar en ello un mayor control, generando así entre los alumnos las conversas". (JH)

Finalmente, enfrenta la presencia de un contexto donde la evaluación, que inicialmente entiende como regulación del aprendizaje, juega el rol de calificación y valor de cambio.

"La evaluación no opera como proceso hacia los estudiantes, se plantea como calificación y control, no como espacio de reflexión frente a mi rol docente". (AH)

En definitiva, logra observar que gran parte de estas dificultades se presentan muy desbordantes y abrumadoras, pues su visión de la docencia es incompleta, no logra calibrar aún cómo se concatenan, en el contexto real, todas las variables que configuran el proceso de enseñanza - aprendizaje.

\section{INSTITUCIONALES}

En el plano institucional se reconoce la presencia de una cultura escolar y determinadas prácticas que dificultan el trabajo docente. Una práctica recurrente enfrentada son las interrupciones al proceso de enseñanza - aprendizaje y al espacio del aula, lo que obliga a estar permanentemente retomando las actividades, lo cual afecta el ambiente de aula y las posibilidades de aprendizaje.

"Durante el desarrollo de las clases las interrupciones obligan a estar retomando permanentemente el trabajo, y para los estudiantes y para uno se transforma en una complejidad". (NI)

Los patrones de actuación de los colegios, en términos de no permitir cambios metodológicos emergen como una dificultad que enfrentan los sujetos entrevistados, y que los circunscribe a esquemas tradicionales de actuación, con escaso foco en el aprendizaje y las necesidades de sus estudiantes de aula. Desde su perspectiva, se anota:

"Siempre suceden eventualidades que restan tiempo a la realización de una clase idónea, uno como profesor tiene muchas estrategias didácticas que anhela aplicar, pero el colegio y a veces el docente guía, imponen sus metodologías uno termina replicando las mismas estrategias dadas por ellos, sin poder innovar". (KC)

Se enfrenta una cultura escolar, reflejada en los juicios y acciones de docentes guías que no favorece la innovación o el intentar dar respuesta a los requerimientos reales del estudiantado. Cultura escolar que, además, les enfrenta a una diversidad de profesorado, en términos de perfiles y exigencias, en el que predomina, esencialmente, un corte tradicional. Las siguientes respuestas lo dejan demostrado:

"Las metodologías impuestas por los colegios, ya que tienen su propia mirada de la educación que quieren entregar, la que en ocasiones no es la correcta para el contexto en el cual se encuentran, el pensamiento de los propios profesores de los centros educativos, basado sólo en la entrega de conocimientos como un mero trámite olvidándose de los valores, de la enseñanza para la vida”. (CC)

"Los profesores son muy diferentes en sus perfiles y exigencias para el trabajo en aula predominan aquellos que no están centrados en mejorar, sino más bien en replicar lo que han hecho siempre, les cuesta atender a la diversidad de los alumnos, y en ocasiones pienso que ni siquiera se dan cuenta de que los alumnos de hoy son distintos”. (RC)

Esta cultura escolar se traduce en prácticas pedagógicas centradas en el uso excesivo de la guía, la reproducción y el traspaso de información, pues sigue patrones de actuación preestablecidos. Así lo plantea la siguiente respuesta:

"El abuso que uno tiende a hacer de una sola metodología de enseñanza para trabajar con los estudiantes, se innova poco, debido también a la carencia de tiempo y a la costumbre y hábitos del profesor, por lo que el proceso se torna monótono no sólo para los alumnos, sino también para uno, que es quien enseña y trabaja con los estudiantes". (JH)

Una herramienta que visualiza como una amenaza para el trabajo la constituye la evaluación, que finalmente consagra formas de enseñar y aprender distanciadas del aprendizaje y su retroalimentación, redundando en el escaso impacto que se logra en términos de aprendizaje. Así: 
"Gran parte de lo que los alumnos aprenden en clase resulta poco significativo para ellos, por lo tanto, se desmotivan uno ve a docentes que en realidad no tenían interés en que sus alumnos aprendieran, solo es aprobar por aprobar, creo que las calificaciones no reflejan aprendizaje, por lo tanto los estudiantes se mueven por la nota". $(\mathrm{OH})$

Es consciente de que se trata de un trabajo que demanda mucho del profesorado, y que ve en los tiempos disponibles una fuente importante de tensión, si lo que se pretende es responder satisfactoriamente a las demandas planteadas. En ese sentido, plantea:

"Los tiempos, me he dado cuenta que para realizar el trabajo de una forma que me deje realmente satisfecha, es decir, para hacer bien la pega necesito tiempo, necesito tiempo para planificar, revisar, crear, retroalimentar y además para estudiar. Otra dificultad es la necesidad de aprendizaje, tengo mucho que aprender y a veces me asusta el hecho de sentir que si no lo hago me pueda convertir en una docente mediocre". (IC)

\section{PERFIL DEL ALUMNADO}

Otro aspecto que tensiona y genera dificultades a este profesorado en formación refiere a los elementos del perfil estudiantil, es decir, del sujeto al cual es preciso atender. Así lo refleja el siguiente relato:

"La nueva idiosincrasia de los jóvenes hoy día, los patrones de comportamiento y valoración transmitidos por los medios de comunicación que los hacen comportarse de manera estereotipada, imitada de sus ídolos juveniles como lo son los cantantes de reguetón o de cualquier género que atente contra una postura de humildad, de desarrollo, de consciencia, empatía o valores propios. No sé aun a ciencia cierta si estos jóvenes actúan autónoma o heterónomamente, pero en su mayoría elegiría la segunda opción”. (GC)

Se trata de estudiantes que tensionan el encuentro didáctico, cuyo comportamiento en el aula suele estar atravesado por las particularidades de los contextos socioculturales de origen. Así lo muestra el siguiente relato:

"Los problemas de comportamiento de los estudiantes, los alumnos tienen dificultades para atender y mantenerse concentrados en una tarea, se distraen con facilidad, sus contextos de origen no facilitan el desarrollo de la clase y lo que en definitiva aprenden". (NI)

En consecuencia, declara sentirse muy expuesto al requerimiento de atender a la diversidad de estudiantes presentes en el aula, en términos de sus necesidades, intereses y estilos de aprendizaje, lo cual condiciona la gestión que se hace de la docencia; problemática altamente referida en las entrevistas:

"Otro obstáculo fue buscar herramientas que me permitieran lograr motivar a los estudiantes para lograr aprendizajes, ya que pude ver un gran desinterés por los aprendizajes y además están rodeados de otros elementos más atractivos, como por ejemplo los celulares y todo lo que en ellos pueden portar". (MJC)

"Responder a la diversidad de estudiantes en el aula, en ocasiones se torna complejo manejar los tiempos de la clase, pues la disposición de los estudiantes en variada”. (PC)

“Los estudiantes son muy heterogéneos y resulta difícil responder a su diversidad de intereses”. (RC)

\section{FORMACIÓN}

Advierte que este proceso de convertirse en docentes, se encuentra mediado por el conjunto de experiencias preformativas que han tenido en su proceso de escolarización, y que se manifiestan en sus actuaciones con más frecuencia de la que pudieran imaginar. Así lo afirman:

"Y creo que el peso de mis 12 años de escolaridad me jugaron también en contra, ya que a veces me pillaba realizando cosas que sabía que no son las más esperadas”. (VC)

Constata, además, que su incorporación al aula, la asume sin el suficiente acompañamiento, resultando una tarea más compleja aún al experimentar esta suerte de sentimiento de desprotección.

Uno de los obstáculos es la sobrecarga de deberes que tenemos con la práctica, en el establecimiento se encuentra la poca ayuda u orientación que muchas veces se tiene del profesor guía, ya que éste en ocasiones ve a los practicantes como alguien 
que ya debería manejarse en su ejercicio docente y, por lo tanto, se le exige como si ya fuese un profesor. El principal obstáculo es que muchas veces los alumnos en práctica nos encontramos frente a grandes exigencias por parte del colegio y para cumplirlas debemos actuar según nuestros propios conocimientos, experiencias anteriores e intuiciones, ya que muchas veces no contamos con alguien que nos guie de la forma esperada”. (AC)

Una de las consecuencias que advierte, de lo anterior, es terminar replicando, en los espacios de aula, modelos que no están pensados en el alumnado y sus necesidades de aprendizaje. A ello refiere el siguiente relato:

"Uno como profesor tiene muchas estrategias didácticas que anhela aplicar, pero el colegio y a veces el docente guía, imponen sus metodologías y uno termina replicando las mismas estrategias dadas por ellos, sin poder innovar". (KC)

\section{SOCIALES}

En un plano más general, es consciente de las limitaciones sociales que enfrenta la docencia, representadas por la existencia de una sociedad con olvido de valores; y el influjo de creencias y modelos instalados que cuesta tensionar y remover. En sus expresiones refleja lo siguiente:

"El pensamiento de los propios profesores de los centros educativos, basado sólo en la entrega de conocimientos como un mero trámite, olvidándose de los valores, de la enseñanza para la vida”. (CC)

"En primer lugar no trabajo solo y por lo mismo la persona que está a cargo de mi práctica tiene arraigada ciertas creencias que muchas veces difieren de las mías, por lo que al momento de enseñar siento que yo no soy el cien por ciento como profesor, no aplico ni la metodología que más me adecúa ni tampoco la técnica de enseñanza que considero más adecuada”. (EH)

En definitiva, enfrentados al ejercicio de identificar sus obstáculos, problemas o limitaciones, el profesorado en formación pone de manifiesto las distintas facetas que configuran el trabajo docente, el que se define desde las interacciones con un alumnado diverso para conseguir su participación en su proceso de formación y atender sus distintas necesidades. Todo ello, en un contexto -el escolar- caracterizado por una serie de rutinas y patrones de actuación que muestran serias dificultades para responder a los variados requerimientos y perfil de su público usuario, en nuestro caso, estudiantes.

\section{Discusión y conclusiones}

El estudio que ha intentado aproximarse cualitativamente a la comprensión de las experiencias pedagógicas desarrolladas por el profesorado en formación en su trayecto a la práctica y al trabajo docente devela tanto el tipo de docente que cada quien es, siente o busca ser, así como los principales obstáculos, problemas o limitaciones a los que se ha debido enfrentar. Este permite concluir la presencia de un modelo de docente, esquema conceptual, figura simbólica, autoridad encarnada que se impone en la idea de construcción que se siente, se busca o se pretende. Se trata de una acción que demanda en lo técnico y relacional, que se encuentra siempre comprometida con la idea de transformación y con una complejidad que lo circunda, dada su naturaleza esencialmente humana.

Por lo tanto, en este grupo de docentes en formación, emergen con fuerza distintas dimensiones asociadas a su rol y a las habilidades necesarias para su desarrollo; al respecto destacan, particularmente, las referidas a la dimensión artesana, reflexiva y social, mediadas, transversalmente, por las de carácter personal.

A partir de esta última, se compromete la idea de un profesorado que, desde la cercanía con el estudiantado, genera respeto, logra comprensión, alcanza involucramiento y consecuencia, y busca, en forma permanente y decidida, constituirse en una tarea que es reconocida como intrínsecamente humana, con toda su complejidad y riqueza.

La complejidad se evidencia cuando se logra reconocer el carácter de artesanía que muestra el trabajo docente, lo que obliga a iniciar una labor centrada en el conocimiento del estudiantado; y en un hacer contextualizado que ha de permitir captar la fibra íntima de cada estudiante, grupo o curso, como condición 
necesaria para el aprendizaje. En esa misma dirección, tomando distancia de perspectivas autoritarias, se demanda una decidida faceta reflexiva, donde la pedagogía busca entender la geografía de los contextos y sus actores, anclada en la autorregulación para lograr un equilibrio dinámico entre las miradas conductistas y constructivistas.

Desde la riqueza y aceptando el desafío de formación para la vida, emerge la dimensión social de la tarea docente, fuertemente vinculada a la necesidad de formar para la participación democrática, en una tarea que debe estar orientada al desarrollo de la responsabilidad social, consciente de un mercado y modelo que gobierna las profesiones, y que explica el surgimiento de resistencias en cuanto formas de contestación social que, en el caso estudiantil, se traduce en desencanto o desinterés por aquello que se le pretende enseñar; y en el caso del profesorado, mediante la prevalencia de modelos pedagógicos muy tradicionales con los cuales se "niega" la posibilidad de responder al nuevo territorio y trama de la formación inicial docente.

Los datos recogidos permiten colegir que todas estas dimensiones asociadas al tipo de docente que se pretende están mediadas por las habilidades o facetas personales del rol, lo cual es altamente correspondiente con el carácter humano de esta profesión y que, a la luz de la complejidad de los contextos, suele ser vinculada a la idea de tarea interactiva, que transforma al personal docente en instrumento de su propio trabajo.

Los hallazgos permiten concluir en este trayecto formativo que los lleva al aula, la existencia y reconocimiento de un "choque de las realidades", el que se traduce en un desfase crucial entre las representaciones subliminales y la primera experiencia. Ello resulta altamente coincidente con lo que, además, reportan los antecedentes teóricos, en tanto "los conocimientos y las imágenes que sustentaban tanto las esperanzas de la profesión como de uno mismo en su práctica no se correspondieran con las situaciones concretas que, día a día, deben afrontarse sobre el terreno" (Baillauques, 2010, p. 66). Desde la perspectiva más práctica, este choque lo enfrenta a una diversidad de problemas que lo lleva a entender que enseñar es ante todo una práctica compleja y cada docente es un personaje central en esta actividad con su estilo, intereses y motivaciones particulares.

Tanto es así que la complejidad de la tarea docente reportada por los datos recogidos se asocia a la presencia de fuentes de tensión externas e internas. En lo externo, la complejidad está representada por la presencia de una cultura escolar, con dificultades para sintonizar con los actores y sus procesos, además de la existencia de modelos que, fuertemente instalados, condicionan el ejercicio de la docencia, haciendo prevalecer un rol asumido por sobre el rol docente deseado.

En lo interno, las dificultades están situadas en lo personal, en el desafío que significa enfrentar la docencia de un grupo de estudiantes que se manifiesta en su diversidad; además, destacan las dificultades asociadas a la presencia de una formación, que traducida en el peso de los doce años de escolaridad, termina por replicar modelos, más que imaginar nuevas formas de desarrollo de la docencia. Es posible advertir que esta complejidad se traduce en respuestas de resistencia o adaptación, por parte de los actores, a las demandas del trabajo docente.

Emerge, así, una temprana conciencia de la complejidad que presenta el trabajo docente en este profesorado en formación, lo cual se explica por las particularidades de un sujeto, denominado estudiante, que manifiesta resistencia al proyecto formativo que se le ofrece, desencadenando una dinámica al interior de este espacio, constituida por tensiones y dilemas, y por negociaciones y estrategias de interacción, agudizadas por el problema del control, que los termina interpelando en lo que cada quien es como persona, en un trabajo que exige tal cual lo señala la revisión bibliográfica "una inversión profunda, tanto desde el punto de vista afectivo como desde el cognitivo, en las relaciones humanas con los alumnos" (Tardif, 2004, p.105). Enfrentado a este escenario de dilemas y demandas, experimenta sus propias crisis, las cuales lo llevan a problematizar/ tensionar sus perspectivas más idealistas, teóricas o normativas de la docencia y el trabajo docente.

Una reflexión más personal, como grupo investigador, permite advertir que el trayecto desde la formación inicial al trabajo docente favorece una certera comprensión de la tarea educativa, en tanto descubre que el trabajo a realizar es tensionado por la presencia simultánea de distintos tipos de acción que le otorgan 
complejidad, lo que obliga a los actores a gestionar un conjunto de interacciones personalizadas con el alumnado para conseguir su participación en su proceso formativo. Constata que estas dificultades son diversas y se ven particularmente afectadas por un ambiente de trabajo que se define por interacciones de naturaleza humana.

En consecuencia, este profesorado en formación despierta a la conciencia respecto a un trabajo donde no solo se trata de "hacer un trabajo", sino ante todo invertir en ese trabajo lo que cada quien es como persona, con lo cual se confirma lo que varios estudios sostienen: Respondiendo a lo planteado desde la teoría, el trabajo docente, por el hecho de abarcar un conjunto de tareas complejas siempre situadas, debe ser enseñado en relación con esta complejidad y por medio de la organización personalizada de los aprendizajes respecto a este conjunto de tareas (Belair, 2010) más aún cuando la práctica muestra importantes ámbitos de indeterminación que exceden los esfuerzos de racionalidad técnica (Schon, 2010) y que necesitan ser atendidos en una formación comprometida con una clara comprensión de la docencia.

Es posible concluir, además, sobre la existencia en los grupos practicantes de un conjunto diverso de imágenes, metáforas o constructos sobre la figura docente, que pone de manifiesto el discurso de la autonomía: desde aquel sujeto que se enfrenta reflexivamente a los problemas de la práctica de la enseñanza; pasando por aquella según la cual construye y sistematiza conocimientos a partir de una actividad esencialmente investigadora; que en su desempeño hace uso de juicios bien informados a través de la reflexión sobre la práctica; que se enfrenta de modo activo y consciente a las problemáticas, buscando vías prácticas de avance o superación de obstáculos; y que, fundamentalmente como un sujeto que actúa en situaciones humanas imprevisibles que le impiden actuar bajo secuencias mecánicas, debe recurrir a su capacidad de creación en situaciones singulares. Sin embargo, cuando refieren las dificultades, obstáculos o limitaciones que han encontrado en su trayecto por la práctica, relevan la evidencia teórica en cuando a "la necesidad de considerar el trabajo docente en relación con sus condiciones y determinaciones sociales, políticas, económicas e ideológicas (Martínez, 1998, p.47)

Desde esos condicionamientos -sociales, institucionales, psicológicos, personales, pedagógicos- que experimenta, y tras la observación del profesorado del sistema, que con sus juicios y acciones evidencia sus propias resistencias a asumir, de forma tan determinista, los condicionamientos políticos e institucionales, constatan las contradicciones entre un marco de actuación tecnicista y la presencia institucional del discurso de la autonomía docente.

Para la institución universitaria, así como para este grupo entrevistado, el formar implica no solo establecer un vínculo con el mundo de la práctica, sino también poner en relación dos lógicas (individual y colectiva) independientes, pero complementarias e íntimamente relacionadas. Desde lo individual, es la persona la que, adscribiéndose a una formación específica, se compromete a adquirir los saberes necesarios al ejercicio de la profesión elegida. Y desde lo colectivo, los saberes que el individuo adquiere son compartidos por otros sujetos, de manera que se configura una red con saberes específicos y que resultan claves para el desarrollo de la identidad (Correa, Gervais y Rittershaussen, 2008).

En un proceso de aprendizaje y desarrollo, el profesorado en formación se ve enfrentado a las decepciones y confusiones frente a los comportamientos imprevistos del alumnado; a las incertidumbres debidas a la inestabilidad tan poco imaginada de las situaciones; a la ambivalencia de los afectos y a una fragilidad inesperada del yo docente. Este, en situación de crisis, percibe por un tiempo el riesgo de diluirse al enfrentarse a la complejidad de un oficio que imaginaba y hubiera querido que fuera fácil. En ese sentido, "el yo docente está desconcertado, piensa en los conocimientos y las habilidades que no tiene, que creía poseer o que no había presentido que fueran pertinentes, necesarios" (Baillauques, 2010, p. 66).

Más específicamente, "para cuando se produce la inmersión en las aguas revueltas de la escuela, de poco sirve el conocimiento acumulado durante la formación inicial, agudizándose las necesidades formativas docentes y para el que - paradójicamente no hay apoyos institucionalizados en nuestro contexto" (Correa, Gervais y Rittershaussen, 2008, p. 86). 
Por otro lado, cuando se piensa en la construcción de la identidad docente, la bibliografía señala que "el maestro, antes de ser identificado y admitido (en la formación y en la clase) por sus esfuerzos, su eficiencia y su eficacia, es un personaje (re) conocido: buscado- encontrado en la experiencia individual y colectiva del pasado escolar" (Baillauques, 2010, p. 62).

Enfrentado a una actividad docente cada vez más exigente en términos de los conocimientos, habilidades específicas y cualidades requeridas, el presente estudio genera perspectivas de diálogo en el contexto regional, para enriquecer la discusión y desarrollo de la formación inicial de docentes, entendiendo que no existen modelos ideales al margen de los contextos o marcos de actuación que regulan el ejercicio de la docencia, por lo que se hace necesario sintonizar los modelos teóricos de formación con los límites y posibilidades que ofrece el aula y la escuela. Por consiguiente, explorar el trayecto recorrido desde la universidad a la escuela, en términos de las comprensiones que van desarrollando de sus experiencias pedagógicas, ciertamente, puede contribuir al desarrollo de una adecuada actuación profesional.

\section{REFERENCIAS}

Ávalos, B. y Matus, C. (2010). La formación inicial docente en Chile desde una óptica internacional. Informe Nacional del Estudio Internacional IEA TEDS-M. Santiago de Chile: Ministerio de Educación.

Baillauques, S. (2010). El trabajo de las representaciones en la formación de los maestros. En L. Paquay, M. Altet, E. Charlier y P. Perrenoud (Coords.), La formación profesional del maestro. Estrategias y competencias (pp. 55-87). México: FCE.

Belair, L. (2010). Enseñar la complejidad del oficio de maestro. En L. Paquay, M. Altet, E. Charlier y P. Perrenoud (Coords.), La formación profesional del maestro. Estrategias y competencias (pp. 88-106) México: FCE.

Carbonneau, M. y Hétu, J. (2010). La formación práctica de los maestros y el nacimiento de una inteligencia profesional. En L. Paquay, M. Altet, E. Charlier, y P. Perrenoud (Coords.), La formación profesional del maestro. Estrategias y competencias (pp. 107-138). México: FCE.

Cisternas, T. (2011). La investigación sobre formación docente en Chile. Territorios explorados e inexplorados. Calidad en la educación, 35, 131-164.

Cornejo, J., Fuentealba, R. (2008). Prácticas reflexivas para la formación profesional docente ¿qué las hace eficaces? Santiago: Ediciones UCSH.

Correa, E., Gervais, C., Rittershaussen, S. (2008). Vers une conceptualisation de la situation de stage: explorations internationales [Hacia una conceptualización de la situación de práctica: exploraciones internacionales]. Montreal: Editions du CRP.

Davini, M. C. (1995). Formación docente en cuestión: Política y pedagogía. Buenos Aires: Paidós.

Diker, G., Terigi, F. (1997). La formación de maestros y profesores: Hoja de ruta. México: Paidós.

Flick, U. (2007). Introducción a la investigación cualitativa. Madrid: Morata

Fullan, M., Hargreaves. A. (1996). La escuela que queremos. B. Aires: Amorrortu

Hernández, R., Fernández, C. y Baptista, M. (2010). Metodología de la investigación (5a ed.). México: McGraw-Hill Imbernón. F. (1994). La formación del profesorado. Buenos Aires: Paidós

Labra, P. (2011). Construcción de conocimiento profesional docente: El caso de la formación en la práctica (Tesis de doctorado). Universidad Academia de Humanismo Cristiano

Latorre, M. (2006). Nuevas miradas, viejos problemas: Las relaciones entre formación inicial y ejercicio profesional docente. Foro Educacional, 10, 41-63

Latorre, M. (2009). Prácticas pedagógicas en la encrucijada: Argumentos, lógicas y razones de los actores educativos. Pensamiento Educativo, 44-45

Martínez, J. (1998). Trabajar en la escuela. Profesorado y reformas en el umbral del siglo XXI. Madrid: Unigraf 
Montecinos, C., Solís, M. C., Contreras, I. y Rittershaussen, S. (2009). Muestras de desempeño docente. Instrumento para evaluar la calidad de la enseñanza y su impacto en el aprendizaje. Santiago: Ediciones UC

Montecinos, C., \& Walker, H. (2010). La colaboración entre los centros de práctica y las carreras de pedagogía. Docencia, 42, 65-73

Montecinos, C., Barrios, C., \& Tapia, M. F. (2011). Relación entre estilos de supervisión durante la práctica profesional y las creencias de autoeficacia de los estudiantes de Pedagogía en Educación Básica. Perspectiva Educacional, Valparaíso, 50(2), 96-122

Nocetti de la Barra, A., Mendoza, M., Contreras, G., Sanhueza, K., \& Herrera, S. (2005). Caracterización de las prácticas iniciales de las carreras de pedagogía en universidades chilenas. Concepción: Universidad San Sebastián - FONIDE

Ortúzar, S., Flores, C., Milesi, C., \& Cox, C. (2009). Aspectos de la formación inicial docente y su influencia en el rendimiento académico de los alumnos. Santiago de Chile: Pontificia Universidad Católica de Chile

Pérez, G. (2007). Investigación cualitativa. Madrid: La Muralla

Ritterhaussen, S., Contreras, I., Suzuki, E., Solís, M. y Valverde, P. (2004). Prácticas iniciales en la formación de educadores. Pensamiento Educativo, 35, 192-215

Rozada, J. (1997). Formarse como profesor. Madrid: Akal

Schon, D. (2010). La formación de profesionales reflexivos: Hacia un nuevo diseño de la enseñanza y el aprendizaje en las profesiones. México: Paidós

Solís, M. C., Núñez, C., Contreras, I., Rittershaussen, S., Montecinos, C., \& Walker, H. (2011). Condiciones de la formación práctica de los futuros profesores. Estudios Pedagógicos, 37(1), 127-147

Tardif, M. (2004). Los saberes docentes y su desarrollo profesional. Madrid: Narcea. Universidad Católica del Maule. (2015). Proyecto FIAC UCM 1102. Talca: Ediciones UCM

\section{Notas}

[1]

Proyecto de investigación financiado por el Plan de Mejoramiento UCM1310 de Formación Inicial de Profesores

CC BY-NC-ND 